\title{
Carrier Plus: A sensor payload for Living With a Star Space Environment Testbed (LWS/SET)
}

\author{
Cheryl J. Marshall ${ }^{* a}$, Steven Moss ${ }^{\mathrm{b}}$, Regan Howard ${ }^{\mathrm{c}}$, Kenneth A. LaBel ${ }^{\mathrm{a}}$, \\ Tom Grycewicz $z^{\mathrm{d}}$, and Janet L. Barth ${ }^{\mathrm{a}}$ Dana Brewer? \\ 2NASA Goddard Space Flight Center, Code 561, Greenbelt, MD, USA 20771 \\ ${ }^{b}$ The Aerospace Corporation, Los Angeles, CA 90009 \\ 'NASA Goddard Space Flight Center, Code 425, Greenbelt, MD 20771 \\ ${ }^{d}$ Defense Threat Reduction Agency, 6801 Telegraph Rd., Alexandria, VA 22310
}

\begin{abstract}
The Defense Threat Reduction Agency (DTRA) and National Aeronautics and Space Administration (NASA) Goddard Space Flight Center are collaborating to develop the Carrier Plus sensor experiment platform as a capability of the Space Environments Testbed (SET). The Space Environment Testbed (SET) provides flight opportunities for technology experiments as part of NASA's Living With a Star (LWS) program. The Carrier Plus will provide new capability to characterize sensor technologies such as state-of-the-art visible focal plane arrays (FPAs) in a natural space radiation environment. The technical objectives include on-orbit validation of recently developed FPA technologies and performance prediction methodologies, as well as characterization of the FPA radiation response to total ionizing dose damage, displacement damage and transients. It is expected that the sensor experiment will carry 4-6 FPAs and associated radiation correlative environment monitors (CEMs) for a 2006-2007 launch. Sensor technology candidates may include n- and p-charge coupled devices (CCDs), active pixel sensors (APS), and hybrid CMOS arrays. The presentation will describe the Carrier Plus goals and objectives, as well as provide details about the architecture and design. More information on the LWS program can be found at http://lws.gsfc.nasa.gov/. Business announcements for LWS/SET and program briefings are posted at http://Iws-set.gsfc.nasa.gov
\end{abstract}

Keywords: Living With a Star, focal plane arrays, space experiment, CCD, APS

\section{INTRODUCTION}

The Carrier Plus sensor platform will fly as a part of the second SET flight currently planned for a 2006-2007 launch. Carrier Plus proposes to enable flight validation of both hardened and unhardened sensor technologies, as well as the validation of ground test protocols and on-orbit performance prediction techniques. Candidate sensor technologies include visible/UV focal plane arrays such as charge-coupled devices (CCDs), active pixel sensors (APS), Si hybrid arrays, and charge injection device arrays.

UV/visible focal plane array technologies have been identified in NASA Origins and Structure and Evolution of the Universe roadmapping efforts as a critical to the NASA Mission yet they are inherently sensitive to radiation. NASA continues to require increasing capabilities for science instruments, pushing the state-of-the-art for signal detectability and precision even in the absence of radiation. For example, the great successes in CCD technology (quantum efficiency close to unity in the visible, negligible dark current, charge transfer efficiency (CTE) $>0.999999$, full well $>10^{6}$ electrons, read noise from $2-5$ electrons RMS) are remarkable. Unfortunately, one must also realize that the greater the performance (and science capability), the greater the sensitivity to radiation. Radiation-induced detector degradation limit the type, precision and/or lifetime of science data collection. As concluded by the UV/Optical working group commissioned by the Office of Space Science (OSS), radiation effects and UV quantum efficiency remain the significant challenges for this technology[1]. The working group also identified CCDs as critical to NASA programs for the foreseeable future and also recognized the importance of developing other Si technologies such as APS, p-CCDs and

\footnotetext{
"Cmarshall2@aol.com; phone 1434 376-3402; fax 1413 215-1428; http://radhome.gsfc.nasa.gov
} 
hybrid detectors. The NASA missions or mission concepts which will be most affected by improvements in UV/optical detectors over the next 15 years include Kepler, Full Astronomical (FAME), SuperNova Acceleration Probe (SNAP), Solar Probe, SPIDR, SUVO, Terrestrial Planet Finder (TPF), etc.

Carrier Plus will be implemented in partnership with the Defense Threat Reduction Agency (DTRA). Several of the sensor arrays technologies of interest to NASA (for example, the p-channel charge couple arrays) are being developed by the military under the auspices of programs that have been in place for several years. DTRA will provide co-funding for the Carrier Plus itself as well as providing technology to fly and ground based radiation testing in support of the flight experiment.

The science goals of Carrier Plus are to:

- Validate ground test based prediction of on-orbit FPA performance.

- Reduce uncertainties in methods used to predict on-orbit sensor performance.

- Provide on-orbit heritage of emerging FPA technologies.

- Enhance understanding of various methods now used to assess CTE behavior in flying instruments.

- Enhance understanding of the on-orbit build-up of hot pixels.

\section{DESCRIPTION OF THE SPACE ENVIRONMENT TESTBED (SET)}

Carrier Plus is a part of the Space Environment Testbed (SET) project [2] which is a complementary element of NASA's new Living With a Star (LWS) Program. This program is designed to help humankind understand and successfully exist with the sun and its variability throughout the solar cycle. The SET element is a series of flight testbeds focused on this variability and its effects on space system technology, as well as crew safety. In particular, the SET aims to improve the engineering approach and/or mitigate the effects of solar variability on spacecraft design and operations. The SET began in FY01 and encompasses a series of flight testbeds with correlative environment measurements. [Is there a target launch date for the first SET?] The SET objectives are:

- To learn how to successfully cope with this solar variance by understanding its effects on technology.

- To validate new technology ground test protocols, performance prediction methods, and environment application models.

\subsection{The natural radiation environment}

The near-Earth natural radiation environment can be divided into two categories: the particles trapped in the Van Allen belts and the transient environment. Figure 1 shows a representation of the environment population. The particles trapped in the near-Earth environment are composed of energetic protons, electrons, and heavy ions. The transient radiation consists of galactic cosmic ray particles and particles from solar events (coronal mass ejections and flares). The cosmic rays have low-level fluxes with energies up to $\mathrm{TeV}$ and include all ions in the periodic table. The solar eruptions produce energetic protons, alpha particles, heavy ions, and electrons. To the first order, all of these particle populations are omnidirectional and isotropic.

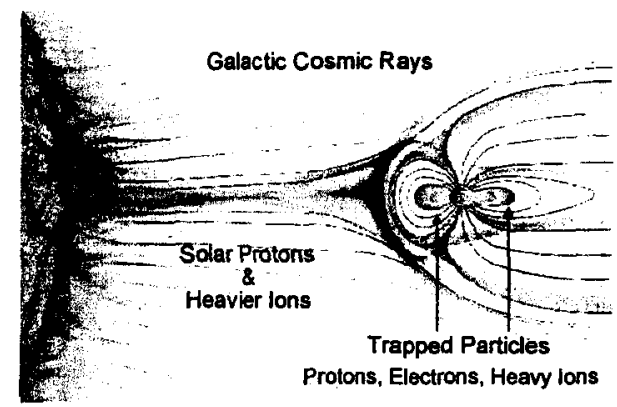

Figure 1: A representation of the radiation environment. From [2]. 
The sun has both a direct and indirect effect on spacecraft technology. Directly, solar events such as solar proton events (SPEs) enhance or modify the background space radiation environment that can affect spacecraft systems and operation. These events may even enhance (pump up) the trapped radiation belts, if they are sufficiently large [1]. The trapped particles pose a significant radiation threat to electronic systems and humans. There are large variations in the level of this hazard, depending on the orbit of the spacecraft, solar activity, and magnetospheric conditions.

The particles from solar events are also a concern for spacecraft designers. In fact, for spacecraft in orbits exposed to these particles, they are often the driver for setting single event effects, total ionizing dose and displacement damage requirements. At this time there is no method for predicting when these events will occur. Warnings have short lead times and are not dependable.

\subsection{The role of space experiment in the qualification of technologies for space}

New sensor technologies with enabling performance characteristics such as increased charge transfer efficiency, reduced read noise or dark current are typically space qualified via a series of ground radiation tests prior to infusion into a critical space system. As we will see in section 3, sensors in space are heavily shielded as a result of their sensitivity to displacement damage effects which introduces additional uncertainties in the ground test protocols, performance prediction methods, and environment application models that the SET program seeks to validate. How one performs performance predictions for flight is based on the existence of ground test data and applicable space environment predictions. If the accuracy of either or both of these factors is improved, there are significant benefits for space system design. The Carrier Plus element of SET provides an opportunity to correlate ground test and on-orbit prediction methods with actual space performance in order to validate the former. Design margins for systems are based on the accuracy of environmental predictions and ground test protocols. By improving the accuracy of both elements, design margins can be significantly reduced, helping to make it possible to space qualify higher performance sensor arrays.

\section{BENEFITS OF THE CARRIER PLUS SPACE EXPERIMENT}

Unlike the majority of microelectronic devices which are primarily vulnerable to total ionizing dose effects, sensors are very susceptible to displacement damage in produced by protons (solar and trapped) as well as secondary particles produced in shielding materials by incoming protons. The models we use to predict displacement damage effects have not been validated by careful comparison with measured on-orbit performance. As a result we have the unfortunate juxtaposition of larger radiation design margins (RDMs) required for a relatively soft technology. Any reduction in the RDM will have a significant positive impact on NASA programs in the process of selecting and qualifying focal plane arrays for various flight programs. A reduction in the RDM may also allow a sensor to be used in a scientifically more desirable orbit (but one with more intense radiation), or for a longer mission time than would otherwise be reasonable. Validated sensor performance models will also permit improved science planning for space missions which is important because it is often the key science measurements that are most affected by the early radiation-induced losses in sensor performance. In addition, as newer technologies such as APS, p-CCDs and Si hybrid arrays are developed, flight heritage provides risk reduction for their use in future programs.

Carrier Plus will permit the validation of ground test based predictions of on-orbit performance. There will be a carefully defined set of on-orbit measurements that correspond exactly with radiation measurements performed on the ground. In addition we will have on-orbit dosimetry and controlled measurement conditions that will allow detailed comparison of predictions with actual performance. Attempts to validate prediction methods in the course of flight programs is difficult because there is generally no on-orbit dosimetry and the on-orbit measurements are only marginally related to the radiation test measurement performed on the ground. For example, the radiation response of CTE is highly application dependent and may be evaluated using radioactive Fe techniques on the ground, yet inferred by cosmic ray tails on orbit. It is very difficult to related these measurements. In fact, one benefit from Carrier Plus will be to provide a wellcontrolled and detailed comparison of various CTE measurement techniques of interest on a given FPA. This will permit one to calibrate the effectiveness of cosmic ray techniques that are more practical in flight, to extended pixel edge response (EPER) and first pixel response (FPR) techniques that are widely used on the ground to evaluate CCDs.

Current programs have experienced a loss of science as a result of radiation induced issues with $C C D$ focal place arrays. For example, Hubble Space Telescope (HST) loses about $10 \%$ of its observing time as a result of radiation-induced hot pixels that necessitate a time consuming anneal to be scheduled monthly. The Space Telescope Imaging Spectrograph 
(STIS), Wide Field Camera 2 (WFC2) and Advanced Camera for Surveys (ACS) instruments aboard HST all employ CCDs that suffer increasing numbers of hot pixels with increasing time on-orbit. CTE degradation is also an important issue. For example, the WFC2 CTE has decreased $15-40 \%$ from 1991-1999, depending on the sky background level. [ref] We currently do not understand the hot pixel annealing mechanism, and note that the measurement suite called for in Carrier Plus may also provide some answers to this troubling radiation effects problem. Finally, we note that it is often the key scientific observations that tend to be adversely impacted first as a result of radiation-induced degradation in the focal plane. The better we can predict the on-orbit performance, the better missions can plan their sequence of scientific observations.

\section{SENSOR MEASUREMENT SUITES PLANNED FOR CARRIER PLUS}

Although the final measurement suite is not as yet determined, Carrier Plus will provide resources required to accurately evaluate the dark current, charge transfer efficiency (if applicable) and transient behavior of the FPAs. Both the average dark current and dark current nonuniformity will be characterized between -20 to $+20^{\circ} \mathrm{C}+/-0.02^{\circ} \mathrm{C}$. Serial subframes will permit the measurement of pixel by pixel dark currents to allow the acquisition of detailed dark current histograms. Temperature dependent CTE measurements employing various techniques such as extended edge pixel response (EPER) [ref], first pixel response (FPR) [ref], and cosmic ray tails [ref] will be provided. It is valuable to assess the CTE using multiple methods in order to validate the relationship between each of these methods which are currently of interest for on-orbit CTE assessment. The temperature control will also permit the CTE measurements to be made at lower temperatures once the CCD dark current (that increases with radiation) becomes too high for measurements to be possible at higher temperatures. Diagnostic signals for the CTE measurements will be provided by dark current integrations, or by an array of LEDs as discussed in section 4. If LEDs are incorporated into the final Carrier Plus implementation then it will also be possible to provide photometric measurements are several wavelengths as well as a quick method for generation of a photon transfer curve [ref]. Proton and heavy ion transients will be characterized by capturing serial subframes of data for analysis. Such measurements may be special interest to focal plane arrays with deep depletion regions such as those employed in $\mathrm{x}$-ray astronomy.

On-orbit dosimetry will be available. TID dosimeters will be mounted near individual FPAs. They will have a resolution of $100 \mathrm{krad}(\mathrm{Si})$ below $50 \mathrm{krad}(\mathrm{Si})$, and a resolution of $1 \mathrm{krad}(\mathrm{Si})$ above $50 \mathrm{krad}(\mathrm{Si})$. The addition of the 4channel proton spectrometer is a current goal and is especially important to the validation of ground test based prediction of on-orbit FPA performance and to the reduction of uncertainties in methods used to predict on-orbit sensor performance. The amount of displacement damage produced by incoming protons (and hence the amount of sensor performance degradation) is very dependent on the proton energy. Hence proton spectroscopy will permit a more accurate test of models that describe the behavior of the focal plane arrays as a function of displacement damage (or time in orbit).

\section{THE CARRIER PLUS ARCHITECTURE}

The Carrier Plus module will be joined to the SET control panel via the command and telemetry lines as indicated in Figure 2, and also derive power from SET. The optical stimulus unit is envisioned to comprise an array of LEDS, possibly at a range of wavelengths. The health of the LEDs will be calibrated using radiation hardened diodes. The core of the thermal management unit is a single stage thermoelectric cooler. Further detail of the FPA Support electronics is shown in Figure 3.

The focal plane array will have bias supplies buffered and trimmed to provide the precise steady bias voltages required by a particular sensor. Special care will also be taken for the clock signals as indicated in the figure. The correlated double sampled readout will be out of one of two radiation tolerant (and spot shielded) analog-to-digital converters (A/D). Signals up to 50,000 electrons will be read out of the "LO" A/D using 10-12 bits for additional resolution whereas larger signals will be read out of the 'HI" A/D. The FPA output will be multiplexed with housekeeping data (including the FPA temperature, voltages, optical stimulus information, etc.) and shipped to the frame processor on the SET motherboard. Although we are not counting on data rates over $1 \mathrm{kbps}$, we expect to have up to $8 \mathrm{Gbit}$ of storage capability and plan to acquire full frame data with lossless compression. Once the orbit for Carrier Plus is finalized, FPA 
shielding will be designed so that measurements taken at 2 week intervals will be sufficient to characterize the radiation induced performance changes.

\section{Optical Sensor Head Block Diagram}

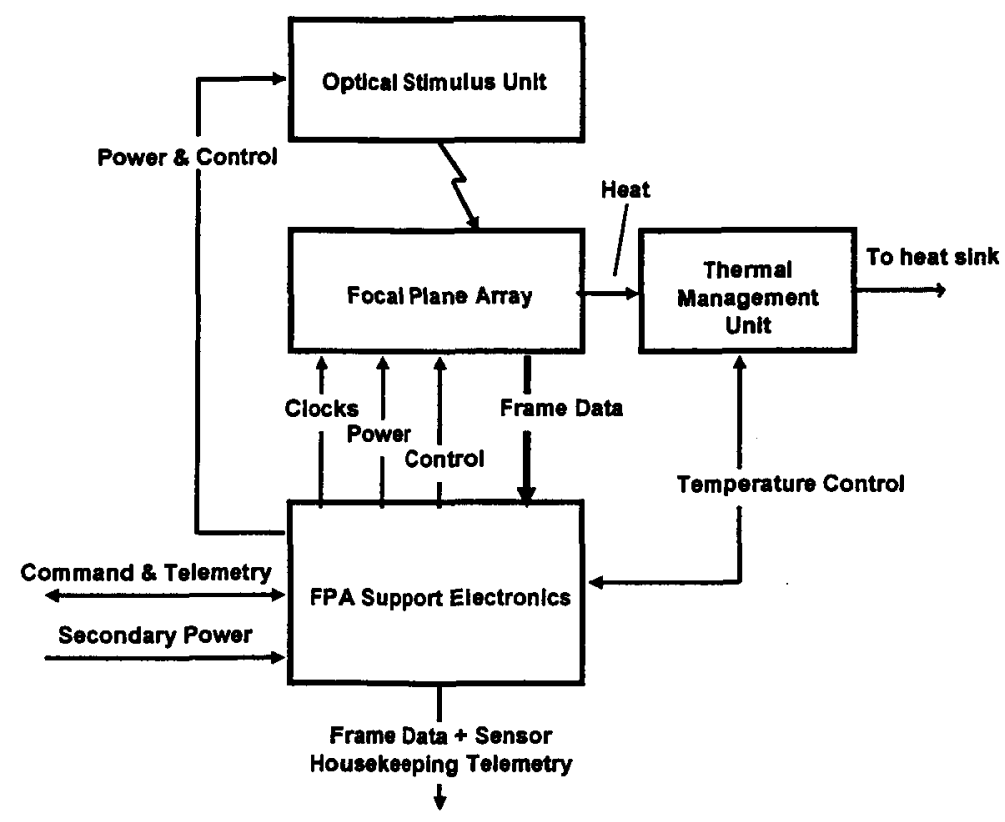

Figure 2: Overview of the Carrier Plus architecture.

The final architecture for Carrier Plus is not finalized. We currently anticipate a single FPA support electronics board for each sensor array. This has the advantage of making the ground testing easier as a board could then be provided to each experimenter. It has not yet been determined whether the optical stimulus unit (assuming it is included in the final design) will employ transmission or reflective optics. Note that given the 2 week interval between measurements it is possible to generate the signals required for a CTE measurements using dark current generation, even for an imager with pA dark currents.

\section{SUMMARY}

The Defense Threat Reduction Agency (DTRA) and NASA Goddard Space Flight Center are collaborating to develop the Carrier Plus sensor experiment platform as a capability of the Space Environments Testbed (SET). The Space Environment Testbed (SET) provides flight opportunities for technology experiments as part of NASA's Living With a Star (LWS) program. More information on the LWS program can be found at http://lws.gsfc.nasa.gov/. Potential experimenters are encouraged to track the business announcements for LWS/SET and program briefings which are posted at http://lws-set.gsfc.nasa.gov. Although NASA will provide funds to help develop a sensor experiment, the accompanying ground radiation test program is the responsibility of the experimenter. It is hoped that the suite of experiments aboard the Carrier Plus space flight will not only provide on-orbit heritage of emerging FPA technologies, but will also validate and reduce uncertainties in current ground test and on-orbit prediction methods. 


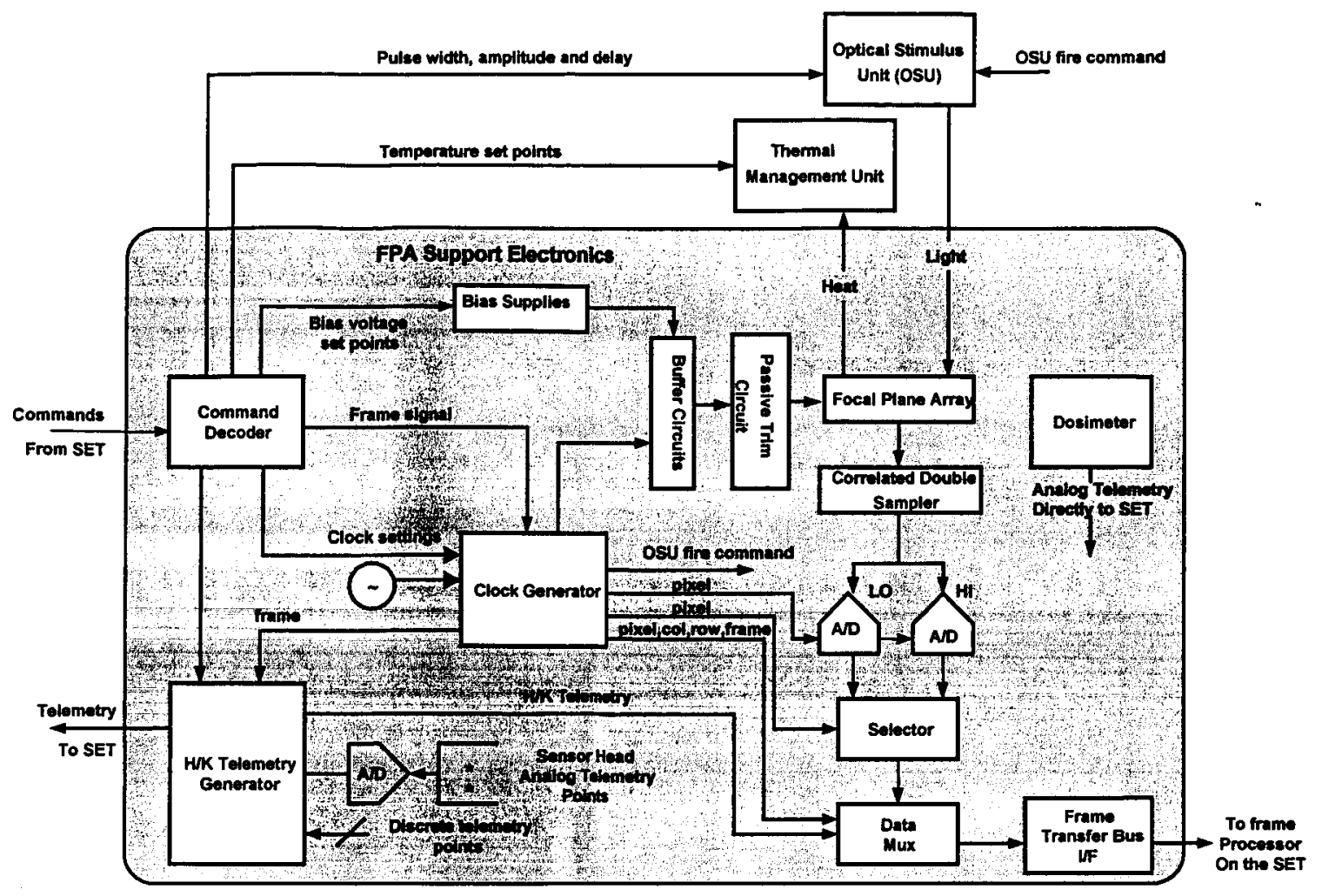

Figure 3: Block diagram of the FPA support electronics

\section{REFERENCES}

1. The working group issued a report entitled, "UV-Optical Detectors for Space Astrophysics," edited by Chris Blades, October 2001.

2. HEART SET reference 


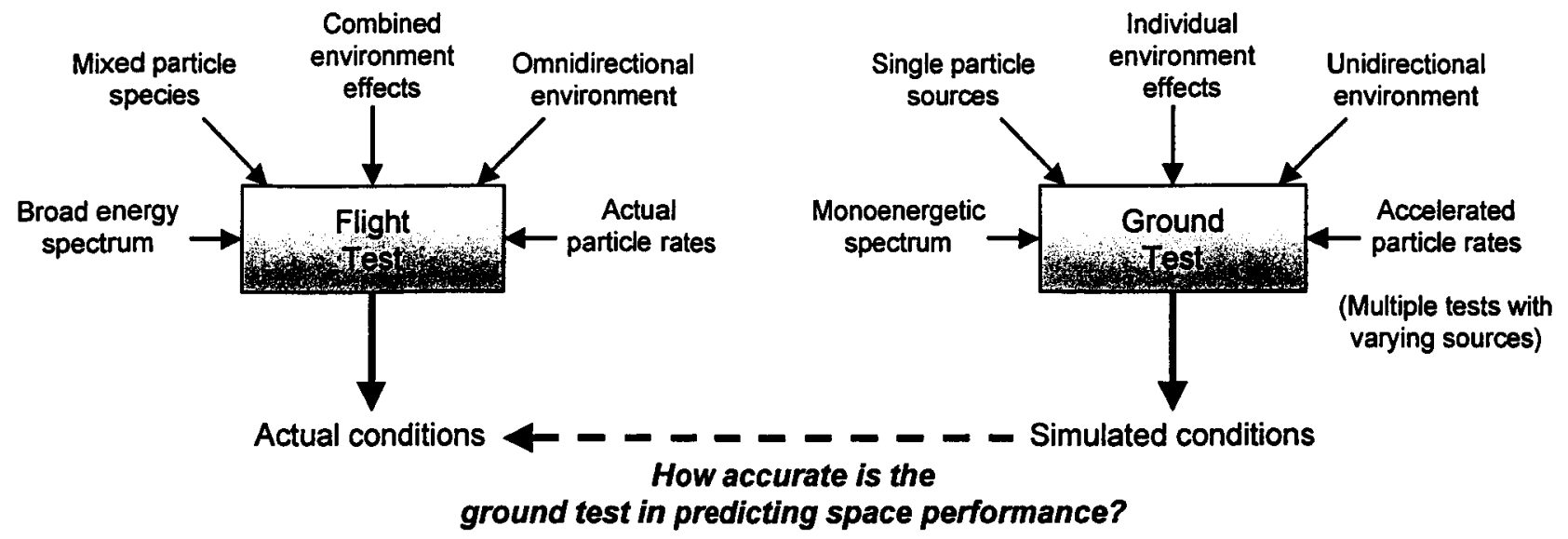

Figure 4: Goal: Improved Ground Radiation Test Protocols 